\title{
An Adolescent Boy with Steroid-responsive Ophthalmoplegic Migraine: A New Case and Systematic Review
}

\author{
(1) Çelebi Kocaoğlu
}

University of Health Sciences, Konya Training and Research Hospital, Clinic of Paediatric Intensive Care, Konya, Turkey

\begin{abstract}
Ophthalmoplegic migraine $(O M)$ is defined as recurrent attacks of headaches with migrainous characteristics, associated with the paresis of one or more ocular cranial nerves and with the absence of any demonstrable intracranial lesion, other than magnetic resonance imaging changes within the affected nerve. In OMs, the most common involvement is seen in the oculomotor nerves. The clinical features of OM including onset in the childhood period, headaches preceding and ipsilateral to the third nerve paresis, dilated pupil and/or ophthalmoplegia. Here, we report on an adolescent boy with OM, treated with steroid and showing a dramatic improvement. It is believed that this case report can add to the understanding and treatment options of OMs.
\end{abstract}

Keywords: Cranial nerve palsy, headache, migraine, ophthalmoplegy

\section{Introduction}

The International Headache Society (IHS) defines ophthalmoplegic migraine (OM) as recurrent attacks of headaches with migrainous characteristics, associated with the paresis of one or more ocular cranial nerves and with the absence of any demonstrable intracranial lesion, other than magnetic resonance imaging (MRI) changes within the affected nerve (1).

In OM, the most common involvement is seen in the oculomotor nerves. The clinical features including onset in the childhood period, headaches preceding and ipsilateral to the third nerve paresis, dilated pupil, ophthalmoplegia that may be permanent and rarely accompanied by aberrant oculomotor regeneration, a minimum of two episodes and a lack of evidence for a structural lesion are known as the criteria for oculomotor OM (2).
In this report, the aim is to present an adolescent boy with $\mathrm{OM}$ involving the third nerve, treated with steroid and showing a dramatic improvement. It is believed that this case report can contribute to the understanding and treatment options of OM.

\section{Case Report}

A 13-year-old boy presented with unilateral (left) ptosis and diplopia with sudden onset and a duration of about 12 hours (Figure 1). The patient had been suffering from such complaints as headache, pain in the left eye, nausea and recurrent vomiting during the previous three days. The patient's history revealed that he had experienced recurrent headache attacks five to six times in the previous six years, and that all episodes had been spontaneously resolved with in two or three days. 


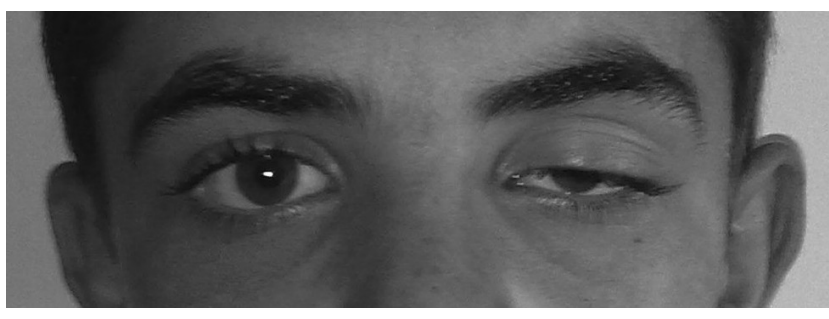

Figure 1. The incomplete ptosis of the left eyelid

However, it was noted that the patient developed eye findings for the first time.

Ophthalmologic examination revealed incomplete ptosis of the left eyelid and an inability to rotate the eye upward, downward, or inward. While normal in the right eye, pupil size was slightly dilated in the left eye. Pupillary constriction was normal in the right eye and sluggish in the left eye. Visual acuity and intraocular pressure in both eyes were within normal limits. Fundus examination showed no abnormal signs such as pallor or edema of the discs. An examination of anterior and posterior segments, as well as macula, vessels and periphery, showed no abnormalities.

Laboratory investigations revealed the following findings: hemoglobin $14.2 \mathrm{~g} / \mathrm{dL}$ : leukocyte count: 6.400/ $\mathrm{mm}^{3}$; and platelet count: $345.000 / \mathrm{mm}^{3}$. C-reactive protein and erythrocyte sedimentation rates were $2.97 \mathrm{mg} / \mathrm{dL}$ and $21 \mathrm{~mm} / \mathrm{h}$, respectively. Serum electrolytes, renal and liver function tests were normal. While brain MRI without contrast was normal, MRI with contrast revealed the diffuse contrast enhancement in the cisternal segment of the left oculomotor nerve. Diffusion MRI also showed a change of blood stream in the left cavernous sinus, suggestive of thrombus. Cerebrospinal fluid examination was normal. No viral agent was identified in the cerebrospinal fluid by polymerase chain reaction.

The patient was diagnosed with $\mathrm{OM}$, based on clinical and laboratory findings. Prednisolone treatment $(2 \mathrm{mg} / \mathrm{kg}$ / day) was started. The recovery in OM was observed on day three of treatment. The case was completely improved one week after the diagnosis. However, steroid treatment was continued for 10 days and then discontinued by tapering off. In order to prevent future attacks, antimigraine prophylaxis (flunarizine) was started, and no recurrence sign was observed during a 12-month follow-up period. The informed consent was taken from the patient's parents for publication.

\section{Discussion}

According to the IHS, OM is diagnosed when at least two or more attacks of migraine-like headaches lasting for 4 days from the onset are accompanied or followed by the paresis of one or more of the third, $4^{\text {th }}$ or $6^{\text {th }}$ cranial nerves. The pathophysiology of OM has yet to be clearly determined. However, different mechanisms, including compressive, ischemic and inflammatory processes, have recently been put forward (1). It is suggested that one or more ocular motor nerves may be compressed by edematous or dilated carotid artery. This is the most accepted theory.

Adult patients with OM often report that OM attacks are experienced at the rate of 1 in 1 or 1 in 2 of all headache attacks they have had. However, in children, OM may be the first manifestation of migraine (3). Therefore, it is usually attributed to aneurysm, trauma, central nervous system infection or immunization. The correct diagnosis is made generally when the clinical condition improves and relapses occur. The majority of the patients experience their initial attack in their first decade, mostly before 5 years of age. Rarely, patients with OM experience their first attacks in adulthood, but they mostly have either a history of typical migraine headaches with or without aura since childhood or a family history of migraine (3). While the onset of migraine attacks in the presented patient was about 7 years of age, the first attack of OM developed in his adolescent period.

Although cranial MRI without contrast is generally normal, approximately $86 \%$ of patients with OM have transient contrast enhancement of the affected cranial nerve in MRI with contrast (1). In this case, MRI with contrast revealed the diffuse contrast enhancement in the cisternal segment of the left oculomotor nerve. Contrastenhanced studies also show that focal thickening takes place at the exit of the nerve in the interpeduncular cistern without enhancement of the cavernous sinus or adjacent dura. Diffusion MRI showed the change of blood stream in the left cavernous sinus which is suggestive of thrombus.

The differential diagnosis of OM includes intracranial aneurysms or tumours, orbital myositis, oculomotor nerve schwannoma, sphenoidalsinus mucoseles, raised intracranial tension with brain herniation and diabetic neuropathy $(3,4)$. The diagnosis of these disorders was excluded through MRI. The fact that the previous history of recurrent headaches resolving spontaneously and other neurological examinations being normal also was supportive of an OM diagnosis.

Optimal treatment for OM still remains unclear. However, prophylactic medications, such as beta blockers and calcium channel blockers, have been proposed as a treatment. Most cases recover completely within days to 
weeks (median time three weeks), but a minority are left with a persistent neurologic deficit $(5,6)$. Some authors believe that immediate administration of steroids at the onset of an attack might reduce permanent sequelae of $\mathrm{OM}$, including residual weakness of the third cranial nerve and pupillary dysfunction (7).

In conclusion, further studies are needed to determine the underlying mechanism and management of this unusual condition. This case met the IHS criteria for OM, and systemic steroid therapy showed promising results. Thus, it is believed that this report can add to the understanding and treatment options of OM.

\section{Ethics}

Informed Consent: The informed consent was taken from the patient's parents for publication.

Peer-review: External and internal peer-reviewed.

Financial Disclosure: The author declared that this study received no financial support.

\section{References}

1. Arasho BD. Ophthalmoplegic migraine in a 15-year-old Ethiopian: case report and literature review. I Headache Pain 2009; 10:45-9.

2. Hansen SL, Borelli-Møller L, Strange P, Nielsen BM, Olesen J. Ophthalmoplegic migraine: diagnostic criteria, incidence of hospitalization and possible etiology. Acta Neurol Scand 1990;81:54-60.

3. Chakravarty A, Mukherjee A. Ophthalmoplegic migraine: A critical analysis and a new proposal. Ann Indian Acad Neurol 2012;15(Suppl 1):S2-6.

4. Roy M, Ghosh J, Deb S, Pandit N. Childhood steroid-responsive ophthalmoplegic migraine. J Pediatr Neurosci 2011;6:69-71.

5. McMillan HJ, Keene DL, Jacob P, Humphreys P. Ophthalmoplegic migraine: inflammatory neuropathy with secondary migraine? Can I Neurol Sci 2007;34:349-55.

6. Bharucha DX, Campbell TB, Valencia I, Hardison HH, Kothare SV. MRI findings in pediatric ophthalmoplegic migraine: a case report and literature review. Pediatr Neurol 2007;37:59-63.

7. Gelfand AA, Gelfand IM, Prabakhar P, Goadsby PJ. Ophthalmoplegic "migraine" or recurrent ophthalmoplegic cranial neuropathy: new cases and a systematic review. J Child Neurol 2012;27:759-66. 